\title{
Reward-Based Learning as a Function of Severity of Substance Abuse Risk in Drug-Naïve Youth with ADHD
}

\author{
Muhammad A. Parvaz, PhD, ${ }^{1,2}$ Kristen Kim, AB, Sean Froudist-Walsh, PhD, \\ Jeffrey $\mathrm{H}$. Newcorn, MD, ${ }^{1,5}$ and lliyan Ivanov, $\mathrm{MD}^{1}$
}

\begin{abstract}
Objective: Attention-deficit/hyperactivity disorder (ADHD) is associated with elevated risk for later development of substance use disorders (SUD), specifically because youth with ADHD, similar to individuals with SUD, exhibit deficits in learning abilities and reward processing. Another known risk factor for SUD is familial history of substance dependence. Youth with familial SUD history show reward processing deficits, higher prevalence of externalizing disorders, and higher impulsivity scores. Thus, the main objective of this proof-of-concept study is to investigate whether risk loading (ADHD and parental substance use) for developing SUD in drug-naïve youth impacts reward-related learning.

Methods: Forty-one drug-naïve youth, stratified into three groups: Healthy Controls (HC, $n=13$; neither ADHD nor parental SUD), Low Risk (LR, $n=13$; ADHD only), and High Risk (HR, $n=15$; ADHD and parental SUD), performed a novel Anticipation, Conflict, and Reward (ACR) task. In addition to conventional reaction time (RT) and accuracy analyses, we analyzed computational variables including learning rates and assessed the influence of learned predictions of reward probability and stimulus congruency on RT.

Results: The multivariate ANOVA on learning rate, congruence, and prediction revealed a significant main Group effect across these variables $[F(3,37)=3.79, p=0.018]$. There were significant linear effects for learning rate (Contrast Estimate $=0.181, p=0.038$ ) and the influence of stimulus congruency on RTs (Contrast Estimate $=1.16, p=0.017)$. Post hoc comparisons revealed that HR youth showed the most significant deficits in accuracy and learning rates, while stimulus congruency had a lower impact on RTs in this group. LR youth showed scores between those of the HC and HR youth.

Conclusion: These preliminary results suggest that deficits in learning and in adjusting to task difficulty are a function of increasing risk loading for SUD in drug-naïve youth. These results also highlight the importance of developing and applying computational models to study intricate details in behavior that typical analytic methodology may not be sensitive to.
\end{abstract}

Keywords: attention-deficit/hyperactivity disorder, substance use disorder, learning, risk, computational analysis

\section{Introduction}

S EVERAL LINES OF RESEARCH have shown that childhood attention-deficit/hyperactivity disorder (ADHD) is associated with elevated risk for later substance use disorders (SUD). A recent meta-analysis documented significantly increased risk for later SUD among children with ADHD (Groenman et al. 2017), and similar results were shown in a prospective study (Molina and Pelham 2003). Others have estimated that lifetime risk for SUD is $\sim 52 \%$ for childhood ADHD that persists into adulthood (Biederman et al. 1995). Longitudinal studies of children with ADHD generally suggest moderate risk for substance abuse (Molina and
Pelham 2014). In addition, a large-scale cross-sectional study of adolescents receiving treatment for SUD found that approximately one-half met criteria for ADHD (Chan et al. 2008).

Some have also argued that SUD risk in youth with ADHD is exacerbated by the presence of comorbid disruptive behavior or mood disorders and/or family history of SUD (Yule et al. 2013; Ali et al. 2016; Mellentin et al. 2016). Thus, in the presence of ADHD, these additional known risk factors may have an additive effect-in other words, having ADHD and family history of SUD is thought to carry substantially higher probability to develop SUD than having either of these alone. Though it is unclear by which mechanisms and to what extent family history of SUD can further increase risk

Departments of ${ }^{1}$ Psychiatry and ${ }^{2}$ Neuroscience, Icahn School of Medicine at Mount Sinai, New York, New York.

${ }^{3}$ Rutgers New Jersey Medical School, Newark, New Jersey.

${ }^{4}$ Center of Neural Science, New York University, New York, New York.

${ }^{5}$ Department of Pediatrics, Icahn School of Medicine at Mount Sinai, New York, New York. 
for later substance abuse among patients with ADHD, from a clinical standpoint it is prudent to consider individuals who have both ADHD and familial SUD to be at particularly elevated risk. In this context, it is important to identify methods to measure and quantify the severity of SUD risk.

Deficits in learning abilities and reward processing, present in both ADHD and familial SUD, are ideal candidates to be considered for exacerbated risk of developing SUD. Youth with ADHD exhibit deficits in decision making and reward-based learning, evident from reports of impaired reward prediction error processing in children with ADHD (Holroyd et al. 2008), which in turn, compromises their ability to make decisions and learn from previous outcomes (Hauser et al. 2014); similar findings have been reported in adults with ADHD (Ibanez et al. 2012). Further, deficits in reward processing have been found in both youth (van Hemel-Ruiter et al. 2013) and adults (Parvaz et al. 2012, 2015) with substance use behavior, suggesting that such deficits may represent a putative predisposition for SUD development in youth.

Moreover, it has been reported that individuals who had at least one parent with alcohol dependence also show reward processing deficits, higher prevalence of externalizing disorders, and higher impulsivity scores while performing a monetary gambling task (Kamarajan et al. 2015). Thus, it is reasonable to question whether the combination of deficits in aspects of learning from one's own behavior and reward processing represents a possible model for SUD vulnerability in children with ADHD and/or parental SUD. To this end, our group has reported preliminary evidence that children with ADHD and parental SUD (with the combination defined as "high risk" for SUD) may exhibit altered functions in the brain reward system before drug exposure (Ivanov et al. 2012), suggesting that altered reward processing might be a central deficit in such "high risk" youth.

In addition to more conventional methods of study, computational methods have been used to more precisely detect differences among individuals with mental disorders versus unaffected counterparts, including those diagnosed with ADHD. For instance, a recent review discusses how the use of a computational, multilevel approach can serve as a framework for studying neural and behavioral abnormalities in ADHD (Hauser et al. 2016). This review uses Marr's three levels of analysis to demonstrate how the variability of behavior among individuals with ADHD can be explained algorithmically, and how such an analytic approach might relate to catecholamine influences on cortico-striatal loops.

A similar approach can potentially provide information to help identify specific deficits in learning and other cognitive functions that may underpin the development of SUD; however, no studies to date have used computational methods to examine behavioral differences in reward-based learning in relation to ADHD and parental SUD as risk factors for SUD. Moreover, no studies have defined different levels of risk based on the presence and/or severity of known clinical risk factors for later SUD. Establishing different risk levels for SUD among at-risk populations, such as children with ADHD and/or parental SUD, may be helpful in clarifying the target population for early intervention strategies and to ultimately lessen the overall burden of SUD.

Here, we examined the potential utility of computational measures (e.g., prediction error, learning rate, and the estimated effects of stimulus congruency and reward prediction on reaction times [RTs]) for detecting levels of SUD risk, which the conventional behavioral analyses of task performance (e.g., RT and nonresponses to targets) may not be sensitive to. We used a hybrid task of learning, reward processing and cognitive control that includes reward cues and outcomes, and conflict resolution components. We hypothesized that the deficits in conflict resolution and reward processing would be associated with higher risk for SUD.

\section{Methods \\ Participants}

We recruited 41 drug-naïve youth (31 male, 10 female) ranging from 8 to 13 years $($ mean $=10.00, \mathrm{SD}=1.57)$. These subjects were not treated with stimulants and were not exposed to substances of abuse. All participants' legal guardians provided written informed consent and children provided assent. Each family was reimbursed $\$ 100$ for completion of the study. The study was approved by the Institutional Review Board at Icahn School of Medicine at Mount Sinai.

\section{Assessments}

Assessment measures (and the domains being measured) were as follows: (1) Psychiatric history: Kiddie Schedule for Affective Disorders and Schizophrenia (K-SADS) Present and Life Time Version interview, administered to the parent and child separately; (2) ADHD symptom severity: Conners' Parent Rating ScaleRevised, Short Version [CPRS-R; (Conners et al. 1998)]; (3) estimate of full-scale IQ (FSIQ): Matrix Reasoning and Vocabulary subtests of the Wechsler Abbreviated Scale of Intelligence [WASI; (Ryan et al. 2003)]; (4) Parental SUD: a semi-structured interview administered to the custodial or primary parent/caregiver, which asked about past and present substance use for each biological parent. It also queried the type of drug used and the length of abuse when it occurred. When a positive report was elicited, additional questions were asked to determine whether (1) the drug use represented a persistent pattern of behavior, (2) it caused functional impairment, and (3) treatment was deemed necessary. In all cases of reported substance abuse, the reporting parent described the abuse as being a "serious drug problem" and indicated that the affected parent needed "treatment."

Participants were divided into three groups based on risk level (Table 1): Healthy Controls (HC, $n=13$ )-including participants with neither ADHD nor parental SUD, Low Risk (LR, $n=12$ )including participants with ADHD only, and High Risk (HR, $n=15$ )-including participants with both ADHD and parental SUD.

\section{Procedure}

Response times (RT) were recorded as participants performed the Anticipation, Conflict, Reward (ACR) Task, which assesses motor responses to targets as a function of reward anticipation, conflict resolution, and reward outcome. As the behavioral analyses reported here were part of a larger fMRI study of SUD risk, the ACR data were obtained while children were being scanned.

Table 1. Demographic Characteristics of Participants (MEAN \pm SD)

\begin{tabular}{lccc}
\hline & Healthy controls & Low-risk & High-risk \\
\hline $\mathrm{N}$ & 13 & 13 & 15 \\
Age, years & $9.92 \pm 1.44$ & $10.00 \pm 1.48$ & $10.07 \pm 1.87$ \\
Sex (M/F) & $9 / 4$ & $9 / 4$ & $13 / 2$ \\
FSIQ & $109.69 \pm 11.55$ & $106.42 \pm 17.74$ & $96.93 \pm 14.86$ \\
CPRS-R* & $7.20 \pm 13.90$ & $32.00 \pm 9.52$ & $29.70 \pm 10.81$ \\
\hline
\end{tabular}

$* p<0.05$.

FSIQ, full-scale IQ; CPRS-R, Conners' Parent Rating Scale-Revised. 
Although the fMRI findings and accompanying behavioral data have previously been published (Ivanov et al. 2012), the current study presents novel secondary analyses using computational methods, specifically investigating deficits in reinforcement learning and reward processing as a function of SUD risk severity.

The ACR consists of four 6-minute and 20-seconds blocks including 32-trials each. In each trial, participants are first presented with a cue, either nonreward or reward, which is represented by a moneybag with a blank or " $\$$," respectively. Next, they see a target (a right- or left-pointing central airplane), which is flanked by airplanes that are moving in a direction either congruent or incongruent with the direction of target. Participants respond in the direction of the target as quickly as possible.

Finally, participants view a reward outcome: an image of a dollar bill for reward, a grayed-out shape of a dollar bill for nonreward, and a hand grabbing a dollar bill for punishment. The reward outcome, which is determined by the preceding cues and the participant's response, is one of the following: (1) expected reward (reward cues followed by $\$ 1$ win for correct responses), (2) expected nonreward (nonreward cues followed by $\$ 0$ for correct responses), (3) unexpected nonreward (reward cue followed by $\$ 0$ for correct responses), and (4) punishment (either cue followed by $\$ 1$ loss for errors). Trial types were counterbalanced across the cues, targets, and reward outcomes. The ACR task is explained in greater detail in our previously published study (Ivanov et al. 2012). Note that a copy of the ACR task can be obtained by contacting the corresponding author.

\section{Data analysis}

First, RT data were analyzed using a 2 (Reward: Reward, Noreward $) \times 2$ (Congruence: Congruent, Incongruent $) \times 3$ (Group: HC, LR, HR) mixed Analysis of Variance (ANOVA). Between-group differences in the overall task performance (i.e., nonresponses across all trials) were investigated using a One-Way ANOVA.

Subsequently, a computational approach was applied to the same behavioral data. As participants did not always receive the reward suggested by the cue, we assumed that they learned to associate a nonbinary value to both the "\$" and "blank" cues. Therefore, a simple reinforcement learning model (Sutton \& Barto, 1998) was applied for each cue, separately.

$$
V_{t}=V_{t-1}+\alpha\left(R_{t}-V_{t-1}\right)
$$

where $\mathrm{V}_{\mathrm{t}-1}$ represents the estimate of the value of the cue before the current trial, $R_{t}$ represents the (positive, negative, or neutral) reward obtained on the current trial, $\left(\mathrm{R}_{\mathrm{t}}-\mathrm{V}_{\mathrm{t}-1}\right)$ is the reward prediction error, and $\mathrm{V}_{\mathrm{t}}$ is the updated value estimate for the current stimulus following the trial. $\alpha$ represents the learning rate, which lies be- tween zero and one and controls for the number of previous trials that influence learning.

We assumed that both the perceived value of the initial stimulus, and the congruency (or lack thereof) of the flanker stimuli would affect RTs. For each subject, and for each possible learning rate, we fit the following model,

$$
R T=\beta_{0}+\beta_{1} V+\beta_{2} W
$$

where RT is the RT, V is the value estimate of the stimulus on the current trial, and $\mathrm{W}$ is the congruency or incongruency of the flankers. The best model for each subject (determined by loglikelihood) was used to identify the best-fitting learning-rate for that participant. A higher estimated learning rate $(\alpha)$ suggests that participants are quickly updating their estimate of the reward value associated with a cue in association with the most immediate reward outcome and are less likely to consider outcomes of past trials. Conversely, a lower $\alpha$ implies that participants are using a longer history of past trials to estimate the value of each cue and might be less influenced by the most immediate reward outcome.

For each participant, the amount to which stimulus congruency and reward prediction influenced RTs could be estimated with $\beta_{1}$ and $\beta_{2}$ respectively. The degree to which these values differ from zero was summarized using t-statistics. The two t-statistics for each participant were taken to represent the effects of stimulus congruency and reward prediction on RTs, respectively. Lower values of the prediction t-statistic suggest that there is a lower influence of the predicted value of the cue on RTs, perhaps due to the participants not learning the association between stimulus type and reward probability. Similarly, lower values of the congruency t-statistic suggest that there is a lower influence of (in) congruency of the flanker on RTs.

These computed variables (i.e., learning rates, and the estimated effects of stimulus congruency and reward prediction on RTs) were analyzed via a multivariate ANOVA with polynomial contrasts, with Group (Control, LR, HR) as a fixed factor. Post hoc pair-wise group comparisons were conducted using Tukey's honestly significant difference (HSD), which inherently accounts for multiple comparisons.

\section{Results}

Means and standard deviations of the dependent variables, including the RT, task performance (nonresponses), learning rates, and congruence and prediction t-scores are provided in Table 2.

\section{Analyses of conventional variables}

The groups did not differ in age, gender distribution, and FSIQ scores. The 2 (Reward: Reward, No-reward) $\times 2$ (Congruence: Congruent, Incongruent) $\times 3$ (Groups: HC, LR, HR) mixed ANOVA

Table 2. Means and Standard Deviations of Dependent Variables

\begin{tabular}{lccc}
\hline & Controls & Low-risk & High-risk \\
\hline RT: reward congruent (ms) & $628.00 \pm 162.92$ & $592.16 \pm 117.12$ & $582.07 \pm 97.43$ \\
RT: reward incongruent (ms) & $707.96 \pm 154.25$ & $662.74 \pm 123.03$ & $651.18 \pm 115.17$ \\
RT: no reward congruent (ms) & $649.21 \pm 151.18$ & $605.54 \pm 94.98$ & $617.76 \pm 99.43$ \\
RT: no reward incongruent (ms) & $755.01 \pm 150.95$ & $677.01 \pm 151.05$ & $668.88 \pm 113.12$ \\
Nonresponses & $2.31 \pm 4.09$ & $4.31 \pm 5.79$ & $8.20 \pm 7.37$ \\
Learning rate & $0.11 \pm 0.13$ & $0.25 \pm 0.36$ & $0.37 \pm 0.37$ \\
Congruence T-scores & $-3.01 \pm 2.21$ & $-2.30 \pm 1.65$ & $-1.36 \pm 1.28$ \\
Prediction T-scores & $-1.40 \pm 2.03$ & $-0.26 \pm 1.86$ & $-0.95 \pm 2.00$ \\
\hline
\end{tabular}

$\mathrm{RT}$, response time. 


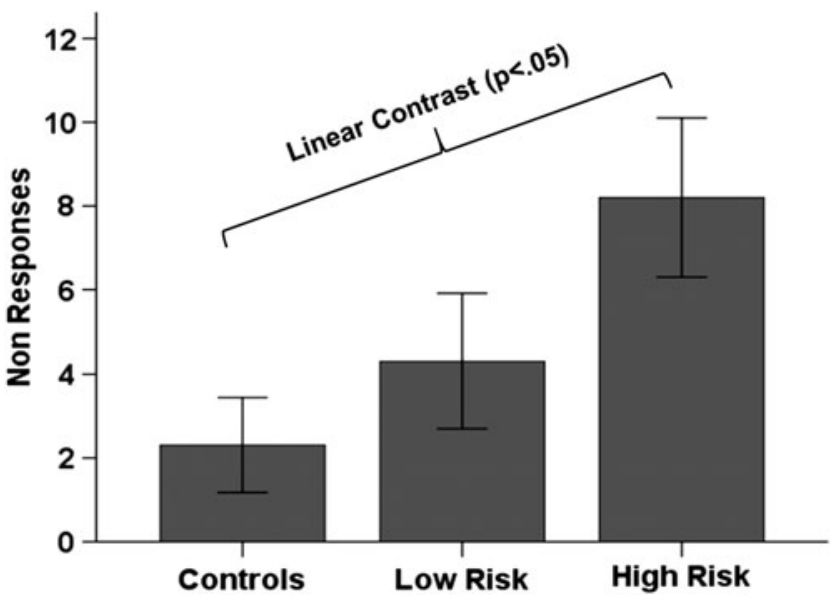

FIG. 1. Bar graph showing a significant group main effect in task performance (i.e., nonresponses). The main effect was driven by a significant difference between Controls and High Risk groups, such that High Risk youth did not respond to significantly more trials compared with Controls. Bars represent the mean and error bars represent the standard error of mean.

for RT revealed a significant main effect of Reward for RT $[\mathrm{F}(1,37)=26.0, p<0.001$; No-reward $>$ Reward; Fig. $1 \mathrm{~A}]$ and Congruence $[\mathrm{F}(1,37)=141.2, p<0.001$; Incongruent $>$ Congruent; Fig. 1B], whereas the Group main effect $(p=0.463)$ and interactions ( $p>0.067)$ did not reach significance.

The One-Way ANOVA on task performance (i.e., nonresponses) showed a significant main Group effect $[F(2,38)=3.53, p=0.039]$, which was driven by a significant difference between HC and HR ( $p=0.013$; HR $>$ Controls; Fig. 2); nonresponses in the LR group did not differ from those in HC and HR groups $(p>0.095)$.

\section{Analysis of computational variables}

The multivariate ANOVA on learning rate, effects of stimulus congruency and reward prediction on RTs revealed a significant main Group effect across these variables $[F(3,37)=3.79$, $p=0.018]$. Closer inspection of each variable revealed significant linear effects in learning rate (Contrast Estimate $=0.181, p=0.038$; Fig. 3A), and in effects of stimulus congruency on RTs (Contrast Estimate $=1.16, p=0.017$; Fig. 3B). Post hoc comparisons, using Tukey's HSD, revealed that these effects were driven by the differences between HC and HR youth (congruence: $p=0.043$, $\mathrm{HR}>\mathrm{HC}$; learning rate: $p=0.093, \mathrm{HR}>\mathrm{HC}$ ). These results show that HR participants showed the most profound performance deficits compared with HC.

\section{Discussion}

The main findings from this proof-of-concept study were that deficits in learning in youth with ADHD were associated with elevated SUD risk status. These findings additionally highlight the relative utility of computational analyses over conventional behavioral analyses in relation to SUD risk.

Conventional analyses brought forth the main effects of reward and congruence, which are expected. All participants responded faster for rewarded and congruent trials compared with trials that were not rewarded and incongruent trials. These findings are useful for establishing the validity and potential utility of the ACR task in indexing process related to reward processing and conflict resolution. However, the comparisons of RT during different components of the task showed no significant difference among groups, and in that sense, have limited value for distinguishing among individuals with different levels of SUD risk. This finding highlights two important points. First, although there are multiple reports showing deficiencies in task performance in individuals with ADHD, general parameters of task performance do not necessarily differ between youth with and without ADHD and may not be specific to ADHD. For example, a meta-analysis demonstrated moderate effect sizes and lack of universality of executive function deficits among individuals with ADHD, which further suggests that executive function weaknesses are neither necessary nor sufficient to cause ADHD but to be one important component (among many) of the complex neuropsychology of ADHD (Willcutt et al. 2005). Second, the identification of individuals who are most likely to
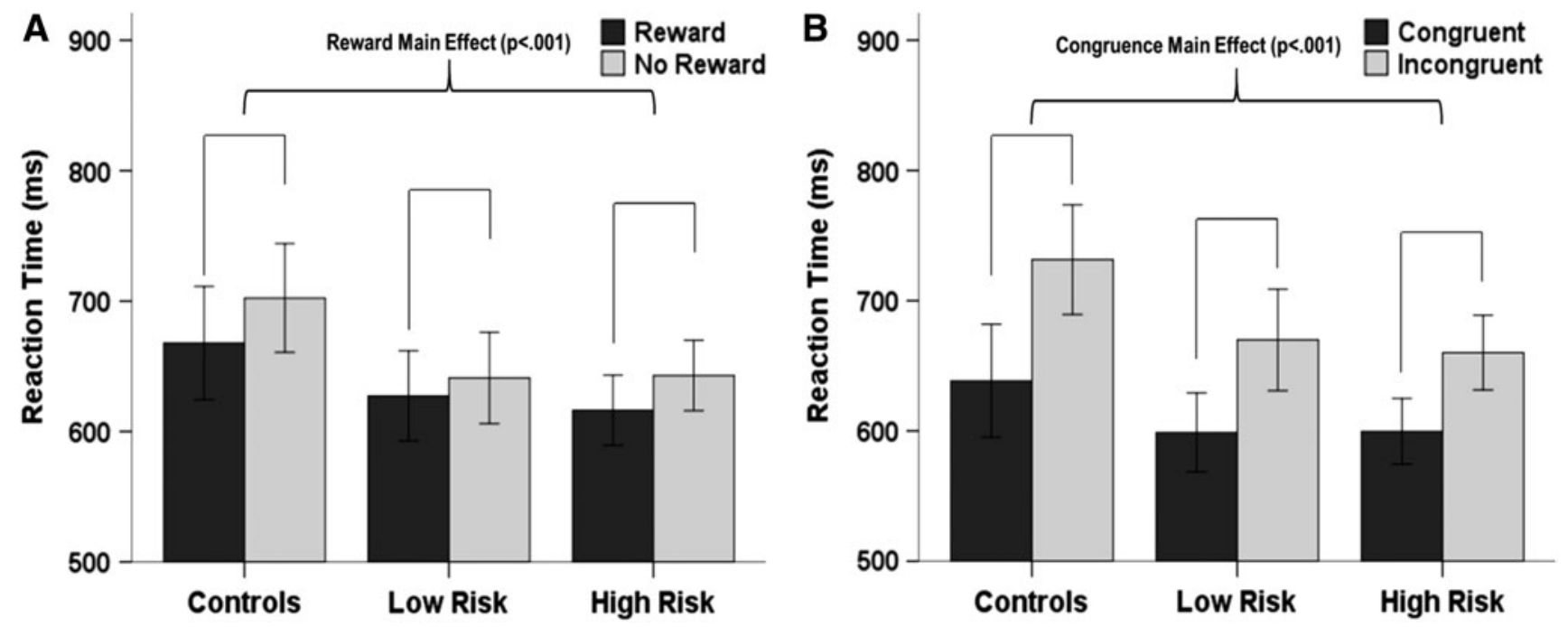

FIG. 2. (A) Main effect of reward, such that participants across all groups responded faster for Reward compared with No Reward condition. (B) Main effect of congruence, such that participants across all groups responded faster for Congruent compared with Incongruent condition. Bars represent the mean and error bars represent the standard error of mean. 

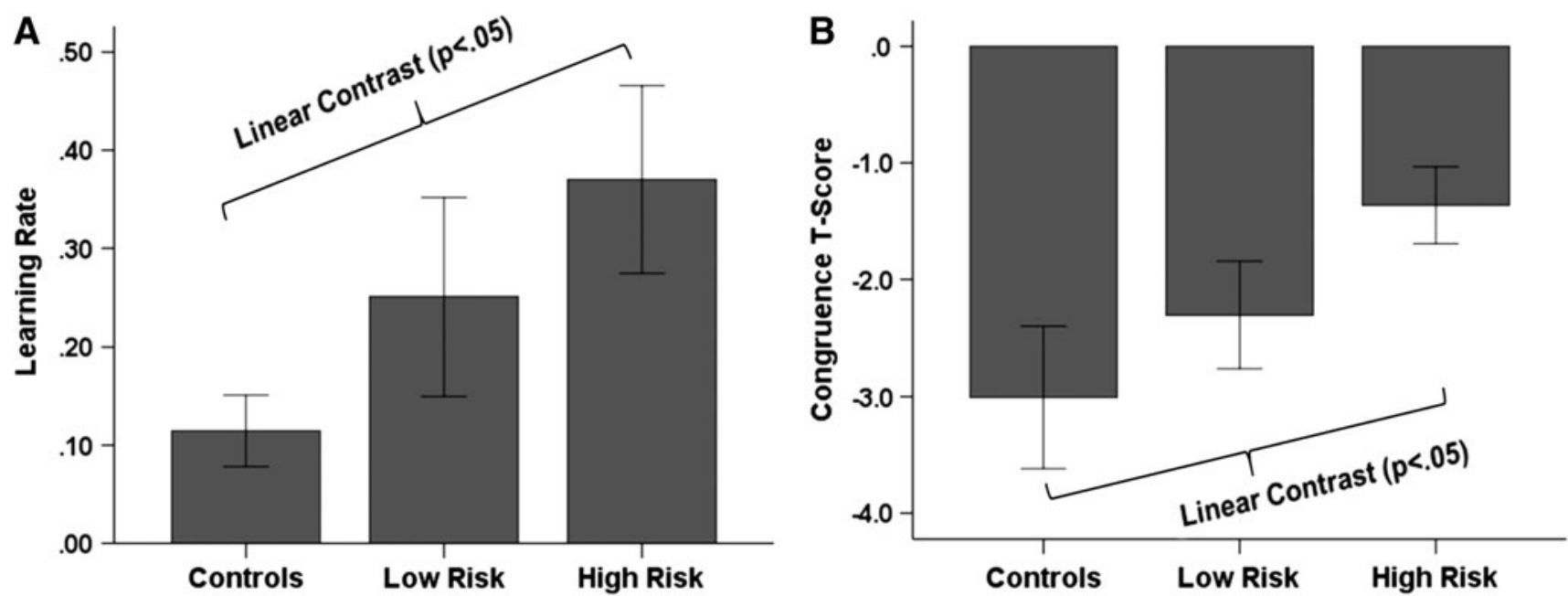

FIG. 3. Significant linear contrasts for (A) Learning Rate, and (B) Congruence, such that both learning rate and congruence varied between groups as a function of risk severity with youth in High Risk group performing worse than those in the Low Risk group, who in turn perform worse than Controls. Bars represent the mean and error bars represent the standard error of mean.

develop subsequent SUD has proven very difficult, even with the application of longitudinal designs (Molina et al. 2013). Multiple reasons may contribute to such unremarkable findings, including the fact that most existing longitudinal studies have sample sizes of $\sim 100$ participants, insufficient to examine the association between risk factors and SUD, which occurs at relatively low frequency even in HR samples (Substance Abuse and Mental Health Services Administration, 2009). Also, longitudinal studies tend to underrecruit HR individuals [e.g., the NIMH-funded Multimodal Treatment of Attention Deficit Hyperactivity Disorder (MTA) study had low rates of comorbid CD (e.g., $8 \%$ ) compared with the $20 \%-25 \%$ figure reported by others] (Molina et al. 2013). Perhaps most importantly, no study has examined the possible differential effects of stimulant treatment and SUD risk in youth with ADHD at relatively HR versus LR for SUD. This is problematic because it leaves open the possibility that a subgroup of youth at elevated risk who may need specialized treatment to prevent later SUD will be overlooked (i.e., findings from this smaller group might not come forward in the larger group analyses). Furthermore, longitudinal studies are fiscally, technically, and practically difficult to carry out, and the results are often plagued by limitations that may preclude definitive conclusions. Therefore, it is imperative for the field to develop methods that can reliably assess individuals at risk at early age and provide reliable results related to their probability for later SUD.

Using computational modeling analyses in this study is a modest step in that direction. Results from these analyses highlight that youth with the highest risk for SUD showed the most profound impairment in task dependent learning (learning rate) and in adapting their behavior based on congruency (congruency t-score), compared with $\mathrm{HC}$ youth, whereas the performance of participants with LR for SUD was in between. Based on this finding, one can speculate that drug-naïve youth with cumulative risk for SUD implement a learning strategy that relies predominantly on the most immediate outcome (as opposed to incorporating information from overall performance during the task, as is the case for $\mathrm{HC}$ subjects). Also HR youth appear to have the most trouble with the resolution of congruency, which suggests the most severe level of impairment of behavioral control, compared with the other two groups. The one exception to this pattern is the prediction error variable, which showed that the two at-risk groups may both have deficits compared with the control group, but do not differ from each other. It is possible that that a more precise and compelling reward paradigm could elicit results that may differentiate between individuals at different risk levels; this remains to be tested. Taken together, our computational results provide preliminary evidence that novel analyses of behavioral data may have the utility to differentiate between levels of SUD risk-in other words, it might be possible to use these methods to measure risk severity, in our case among youth with ADHD. As computational psychiatry has made considerable strides in identifying specific mechanisms and neuronal systems that address the heterogeneity of ADHD (Hauser et al. 2016), the current results represent yet another exploratory effort in that direction.

It is important to note that although this study highlights the utility of computational models to assess risk for SUD development in youth, current results are limited to subgroups of youth with ADHD, with and without familial SUD. However, considering that those without ADHD and/or familial SUD may also go on to develop SUD, future longitudinal studies may apply these computational models on larger samples of non-ADHD youth to assess whether additional subgroups can be identified who present higher propensity of developing SUD. Additionally, although these computational variables are currently derived from behavior on the ACR task, given the general nature of the reinforcement learning model, presumably such a method can be applied to a variety of cognitive tasks that have different reward outcomes and target response components (e.g., flanker stimulus). The potential for wide applicability of these computational models lends more robustness to their outcomes and strengthens their generalizability. Additionally, given the nonspecific nature of the reinforcement learning model, our method can be applied to a variety of cognitive tasks that have different reward outcomes and target response components (e.g., flanker stimulus), so that wider applicability of these computational models can lend more robustness to their outcomes and strengthen their generalizability.

\section{Clinical Significance}

This study is the first to demonstrate that computational methods applied to behavioral data on a task designed to be used during 
functional neuroimaging can be used to differentiate ADHD subphenotypes based on levels of SUD risk. These results further suggest that the application of computational analytic approaches to assess behavior may provide a complementary way to quantify different levels of risk for SUD in larger samples.

Moreover, such an approach opens the possibility that measurements of risk severity at baseline can also be used to measure changes in risk secondary to interventions. For instance, in our sample we detected differences in learning rates and congruency in youth at different levels of SUD risk; one possible hypothesis is that deficits in the ability to learn from one's own performance and to solve congruence can be treated with medications that can potentially improve such deficits. Existing literature provides evidence for detectable differences in cognitive abilities between individuals with and without ADHD (Mullane et al. 2009; Swanson et al. 2011) but there is less convincing evidence for the ability of stimulants to "correct" for such deficits and potentially influence aspects of "real life" functioning such as academic performance (Advokat and Scheithauer 2013). However, recent epidemiologic studies from large data sets $(n=2,993,887)$ have shown that measures of negative outcomes related to SUD decrease when individuals with ADHD receive stimulant treatment vs. when they do not take those meds (Quinn et al. 2017). The challenge is to show that similar changes in SUD risk measures may translate into real reduction of risk for later SUD. This can be most reliably demonstrated in studies that use identified measures to reliably measure SUD risk pre and postinterventions. We believe that this proof-of-concept study provides some initial ideas as to how this could be incorporated into subsequent research studies. Subsequent larger scale validation studies are therefore indicated.

Our results should be viewed in the light of several limitations. The current study design could be improved by adding a group without ADHD but with familial SUD to comprehensively assess the additive or orthogonal effects of these risk factors on reward learning. As this study highlights the utility of computational models to assess risk for SUD development in youth, current results are limited to subgroups of youth with ADHD, with and without familial SUD. We also examined risk for SUD using a crosssectional design and do not have longitudinal data to show that the HR and LR groups will present differences in their subsequent patterns of substance use. However, considering that adolescents without ADHD and/or familial SUD may also go on to develop SUD, future longitudinal studies may apply these computational models to larger samples of non-ADHD youth to assess which subgroups will show higher propensity of developing SUD. Also, the ACR task was not specifically designed to capture prediction error, and that may explain why this variable failed to distinguish between the groups. Importantly, the ACR task was obtained while children were receiving an MRI scan; replication of these findings outside the scanner is required. Lastly, the sample size is relatively small, and more appropriate for an fMRI study than a study designed to assess the utility of a novel instrument. However, we offer the results of this preliminary study here to highlight the potential utility of computational methods to differentiate between individuals at varying levels of risk for SUD.

\section{Conclusions}

This study demonstrates that the presence of familial history of SUD and ADHD in youth exacerbates impairments in rewardbased learning. Specifically, youth with both familial SUD and ADHD exhibit significantly lower learning rates and lower be- havior adaptation to task demands compared with their normally developing counterparts. Additionally, the results also highlight the importance of developing and applying computational models to study intricate details in behavior that may not be detected by conventional analytic methods. While this article provides preliminary evidence of reward learning deficits in youth with multiple risks for developing SUD using computational modeling, more comprehensive studies are required to further delineate phenotypic profiles of each of these risk factors and how they may operate together to target preventive interventions for SUD risk in high-risk youth.

\section{Acknowledgments}

This study was funded by grants from the National Institutes of Health [KL2TR001435 (NCATS) and K01DA043615 (NIDA) to MAP; K23PA00003 (NIDA) and R03DA25796 (NIDA) to II]. The content of this publication is solely the responsibility of the authors and does not necessarily represent the official views of the National Institutes of Health.

\section{Disclosures}

M.A.P., K.K., and S.F.W. declare that they have no competing financial interests. I.I. is a member of the Data Safety Monitoring Committee at Lundbeck. J.H.N. is an advisor/consultant for Akili Interactive, Alcobra, Cingulate Therapeutics, Enzymotec, Ironshore, Kempharm, Lundbeck, NLS, Rhodes, Shire, and Supernus; receives grant support from Enzymotec Ltd. and Shire; and serves on the Data and Safety Monitoring Board for Sunovion Pharmaceuticals, Inc.

\section{References}

Advokat C, Scheithauer M: Attention-deficit hyperactivity disorder (ADHD) stimulant medications as cognitive enhancers. Front Neurosci 7:82, 2013.

Ali MM, Dean D, Jr. Hedden SL: The relationship between parental mental illness and/or substance use disorder on adolescent substance use disorder: Results from a nationally representative survey. Addict Behav 59:35-41, 2016.

Biederman J, Wilens T, Mick E, Milberger S, Spencer TJ, Faraone SV: Psychoactive substance use disorders in adults with attention deficit hyperactivity disorder (ADHD): Effects of ADHD and psychiatric comorbidity. Am J Psychiatry 152:1652-1658, 1995.

Chan YF, Dennis ML, Funk RR: Prevalence and comorbidity of major internalizing and externalizing problems among adolescents and adults presenting to substance abuse treatment. J Subst Abuse Treat 34:14-24, 2008.

Conners CK, Sitarenios G, Parker JD, Epstein JN: The revised Conners' Parent Rating Scale (CPRS-R): Factor structure, reliability, and criterion validity. J Abnorm Child Psychol 26:257-268, 1998.

Groenman AP, Janssen TWP, Oosterlaan J: Childhood psychiatric disorders as risk factor for subsequent substance abuse: A metaanalysis. J Am Acad Child Adolesc Psychiatry 56:556-569, 2017.

Hauser TU, Iannaccone R, Ball J, Mathys C, Brandeis D, Walitza S, Brem S: Role of the medial prefrontal cortex in impaired decision making in juvenile attention-deficit/hyperactivity disorder. JAMA Psychiatry 71:1165-1173, 2014.

Hauser TU, Fiore VG, Moutoussis M, Dolan RJ: Computational psychiatry of ADHD: Neural gain impairments across marrian levels of analysis. Trends Neurosci 39:63-73, 2016.

Holroyd CB, Baker TE, Kerns KA, Müller U: Electrophysiological evidence of atypical motivation and reward processing in children 
with attention-deficit hyperactivity disorder. Neuropsychologia 46: 2234-2242, 2008.

Ibanez A, Cetkovich M, Petroni A, Urquina H, Baez S, Gonzalez-Gadea ML, Kamienkowski JE, Torralva T, Torrente F, Strejilevich S, Teitelbaum J, Hurtado E, Guex R, Melloni M, Lischinsky A, Sigman M, Manes F: The neural basis of decision-making and reward processing in adults with euthymic bipolar disorder or attentiondeficit/hyperactivity disorder (ADHD). PLoS One 7:e37306, 2012.

Ivanov I, Liu X, Shulz K, Fan J, London E, Friston K, Halperin JM, Newcorn JH: Parental substance abuse and function of the motivation and behavioral inhibition systems in drug-naive youth. Psychiatry Res 201:128-135, 2012.

Kamarajan C, Pandey AK, Chorlian DB, Manz N, Stimus AT, Bauer LO, Hesselbrock VM, Schuckit MA, Kuperman S, Kramer J, Porjesz B: Reward processing deficits and impulsivity in highrisk offspring of alcoholics: A study of event-related potentials during a monetary gambling task. Int J Psychophysiol 98:182200, 2015.

Molina BS, Pelham WE, Jr. Childhood predictors of adolescent substance use in a longitudinal study of children with ADHD. J Abnorm Psychol 112:497-507, 2003.

Molina BS, Hinshaw SP, Eugene Arnold L, Swanson JM, Pelham WE, Hechtman L, Hoza B, Epstein JN, Wigal T, Abikoff HB, Greenhill LL, Jensen PS, Wells KC, Vitiello B, Gibbons RD, Howard A, Houck PR, Hur K, Lu B, Marcus S: Adolescent substance use in the multimodal treatment study of attention-deficit/hyperactivity disorder (ADHD) (MTA) as a function of childhood ADHD, random assignment to childhood treatments, and subsequent medication. J Am Acad Child Adolesc Psychiatry 52:250-263, 2013.

Molina BS, Pelham WE, Jr. Attention-deficit/hyperactivity disorder and risk of substance use disorder: Developmental considerations, potential pathways, and opportunities for research. Ann Rev Clin Psychol 10:607-639, 2014.

Mellentin AI, Brink M, Andersen L, Erlangsen A, Stenager E, Bjerregaard LB, Christiansen E: The risk of offspring developing substance use disorders when exposed to one versus two parent(s) with alcohol use disorder: A nationwide, register-based cohort study. J Psychiatr Res 80:52-58, 2016.

Mullane JC, Corkum PV, Klein RM, McLaughlin E: Interference control in children with and without ADHD: A systematic review of Flanker and Simon task performance. Child Neuropsychol 15:321342, 2009.

Parvaz MA, Konova AB, Proudfit GH, Dunning JP, Malaker P, Moeller SJ, Maloney T, Alia-Klein N, Goldstein RZ: Impaired neural response to negative prediction errors in cocaine addiction. $\mathbf{J}$ Neurosci 35:1872-1879, 2015.

Parvaz MA, Maloney T, Moeller SJ, Woicik PA, Alia-Klein N, Telang F, Wang GJ, Squires NK, Volkow ND, Goldstein RZ: Sensitivity to monetary reward is most severely compromised in recently abstaining cocaine addicted individuals: A cross-sectional ERP study. Psychiatry Res 203:75-82, 2012.

Quinn PD, Chang Z, Hur K, Gibbons RD, Lahey BB, Rickert ME, Sjolander A, Lichtenstein P, Larsson H, D'Onofrio BM: ADHD Medication and Substance-Related Problems. Am J Psychiatry 174: 877-885, 2017.

Ryan JJ, Carruthers CA, Miller LJ, Souheaver GT, Gontkovsky ST, Zehr MD: Exploratory factor analysis of the Wechsler Abbreviated Scale of Intelligence (WASI) in adult standardization and clinical samples. Appl Neuropsychol 10:252-256, 2003.

Substance Abuse and Mental Health Services Administration. US Department of Health and Human Services, Center for Substance Abuse Treatment, Rockville, MD, 2009.

Sutton R, Barto A: Reinforcement Learning: An Introduction. MIT Press, Cambridge, MA, 1998.

Swanson J, Baler RD, Volkow ND: Understanding the effects of stimulant medications on cognition in individuals with attention-deficit hyperactivity disorder: A decade of progress. Neuropsychopharmacology 36:207-226, 2011.

van Hemel-Ruiter ME, de Jong PJ, Oldehinkel AJ, Ostafin BD: Reward-related attentional biases and adolescent substance use: The TRAILS study. Psychol Addict Behav 27:142-150, 2013.

Willcutt EG, Doyle AE, Nigg JT, Faraone SV, Pennington BF: Validity of the executive function theory of attention-deficit/hyperactivity disorder: A meta-analytic review. Biol Psychiatry 57:1336-1346, 2005.

Yule AM, Wilens TE, Martelon MK, Simon A, Biederman J: Does exposure to parental substance use disorders increase substance use disorder risk in offspring? A 5-year follow-up study. Am J Addict 22:460-465, 2013.

$$
\begin{array}{r}
\text { Address correspondence to: } \\
\text { Iliyan Ivanov, MD } \\
\text { Department of Psychiatry } \\
\text { Icahn School of Medicine at Mount Sinai } \\
\text { One Gustave L. Levy Place } \\
\text { Box } 1230 \\
\text { New York, NY } 10029
\end{array}
$$

E-mail: iliyan.ivanov@mssm.edu 